\title{
Spotlight on measles 2010: Measles outbreak in Ireland 2009-2010
}

S Gee ${ }^{1}$, S Cotter (suzanne.cotter@hse.ie) ${ }^{1}$, D 0'Flanagan ${ }^{1}$, on behalf of the national incident management team ${ }^{2}$

1. HSE-Health Protection Surveillance Centre, Dublin, Ireland

2. The members of the team are listed at the end of the article.

Citation style for this article:

Citation style for this article: Gee S, Cotter S, O’Flanagan D, on behalf of the national incident management team. Spotlight on measles 2010: Measles outbreak in Ireland 2009-2010. Euro Surveill. 2010;15(9):pii=19500. Available online: http://www.eurosurveillance.org/ViewArticle.aspx?Articleld=19500

This article has been published on 4 March 2010

Measles cases are increasing in Ireland, with 320 cases notified since August 2009. Nearly two-thirds of these cases $(n=206)$ were unvaccinated. In the early stages of the outbreak a substantial number of cases were linked to the Traveller community with some cases also reported among the Roma community, other citizens from eastern Europe and children whose parents objected to vaccination. By February 2010, there had been considerable spread to the general population.

\section{Background}

Measles is a highly infectious disease that can result in serious complications. The only way to prevent infection is through measles vaccination. Measles vaccine was introduced in Ireland in 1985; this was followed by the introduction of the combined measles-mumpsrubella (MMR) vaccine in 1988 for children aged 15 months. In 1992, a second dose of MMR was recommended for all children aged 10 to 14 years. In 1995 , there was a measles and rubella vaccination campaign for children of primary school-age (5-12 years old). In 1999, the age of the second dose of MMR was changed to 4-5 years. In 2002, the age of the first dose of MMR was changed to $12-15$ months, and since 2008 it is recommended at 12 months of age.

In 1985, the year when measles vaccine was introduced, 9,903 measles cases were reported, declining to 201 cases in 1987. However, despite the routine immunisation programme, further major outbreaks have occurred in 1989 (1,248 cases), 1993 (4,328 cases) and 2000 (1,603 cases).

Since the national collation of quarterly $M M R_{1}$ (first dose) immunisation uptake statistics commenced in 1999, the $M M R_{1}$ uptake rate in those aged 24 months has ranged between 69\% (Quarter 4, 2001) and 91\% (Quarter 3, 2009) [1]. While the immunisation uptake rate is below the target rate of $95 \%$, measles outbreaks like the one seen in 2000 [2] will continue to occur. In addition, there are subpopulations in Ireland who are highly susceptible to measles, e.g. those who refuse the MMR vaccine and communities with low uptake of MMR due to social exclusion and disadvantage.

\section{Methods}

Measles figures presented in this report were based on data extracted from the Computerised Infectious Disease Reporting (CIDR) system on 26 February 2010 and are provisional. Incidence rates were calculated based on population data taken from the 2006 census. Crude area rates and numbers of notified cases are shown according to the eight Health Service Executive (HSE) Area Departments of Public Health.

Case classifications are assigned to notifications in Ireland as per the Case Definitions for Notifiable Diseases [3].

The measles case definition is as follows:

Clinical description: Clinical picture compatible with measles i.e. a generalised erythematous rash lasting for more than three days and a temperature over $38^{\circ} \mathrm{C}$ and one or more of the following: cough, coryza (rhinitis), Koplik's spots or conjunctivitis.

Laboratory criteria for diagnosis are one of the following:

- Detection of measles IgM antibody in the absence of recent vaccination,

- Fourfold or higher rise in measles IgG antibody level in the absence of recent vaccination

- Detection of measles virus (not vaccine strains) in a clinical specimen.

\section{Case classification:}

- Possible: clinically compatible cases,

- Confirmed: a case that is laboratory-confirmed or a clinically compatible case which is epidemiologically linked to a confirmed case. A laboratory-confirmed case does not need to meet the clinical case definition.

A measles case is epidemiologically linked if there was exposure to a laboratory-confirmed case during the infectious period (four days before to four days after rash onset) and this exposure occurred within the expected incubation period of the case under investigation, 7 to 18 days (mean 14 days) before rash onset. 


\section{Epidemiology}

In week 31 in 2009 (week ending 8 August 2009), a confirmed measles case, in an adult who worked in

\section{FIGURE 1}

Number of notified measles cases and crude incidence rate per 100,000 population in the eight Health Service Executive Area Departments of Public Health, Ireland, week 31, 2009 to week 7, 2010

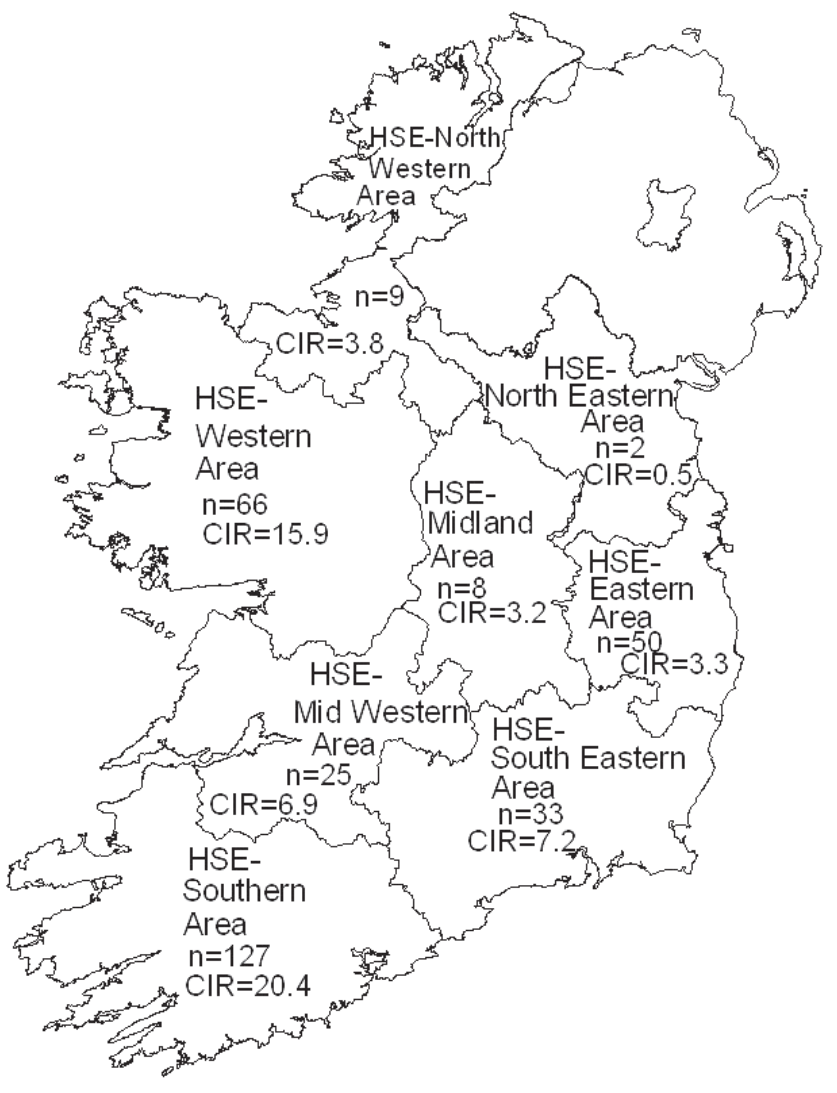

CIR: crude incidence rate; HSE: Health Services Executive. a general practice, was notified in the HSE-Southern Area (Figure 1 shows this location).

In week 33 in 2009, a measles case in a Roma child was notified in the same Area, this case's general practitioner (GP) worked in the same building as the previous case. In week 37, 2009, two measles cases, one in a child from the Traveller community (an indigenous minority group many of whom maintain a nomadic way of life [4]) and one in a hospital contact of this case, were notified in the HSE Southern Area. During weeks 38 and 39, six cases in Travellers were notified in the HSE-Southern Area. From then on measles continued to circulate and spread to other HSE Areas.

Although ethnicity is not routinely collected as part of notification data and may be difficult to establish and report on, it was evident in the early stages of the outbreak that a substantial number of cases were linked to the Traveller community (anecdotal reports). By December, verbal reports from the HSE Southern Area highlighted transmission was now also among children whose parents objected to vaccination, either for perceived safety reasons or for philosophical reasons. During the course of the outbreak a small number of cases were also reported, in different HSE Areas, among the Roma community and other citizens from Eastern Europe. By February 2010, there was considerable spread to the general population.

Measles notifications from 2008 to week 7 of 2010 are shown in Figure 2. During weeks 1-30, 200943 measles cases were notified. In contrast, 320 measles cases were notified between week 31, 2009 and week 7, 2010 (outbreak period to date).

Of the 320 cases notified, 227 (71\%) were classified as confirmed and 92 (29\%) were classified as possible,

\section{FIGURE 2}

Measles notifications by week, Ireland, week 1, 2008 to week 7, 2010

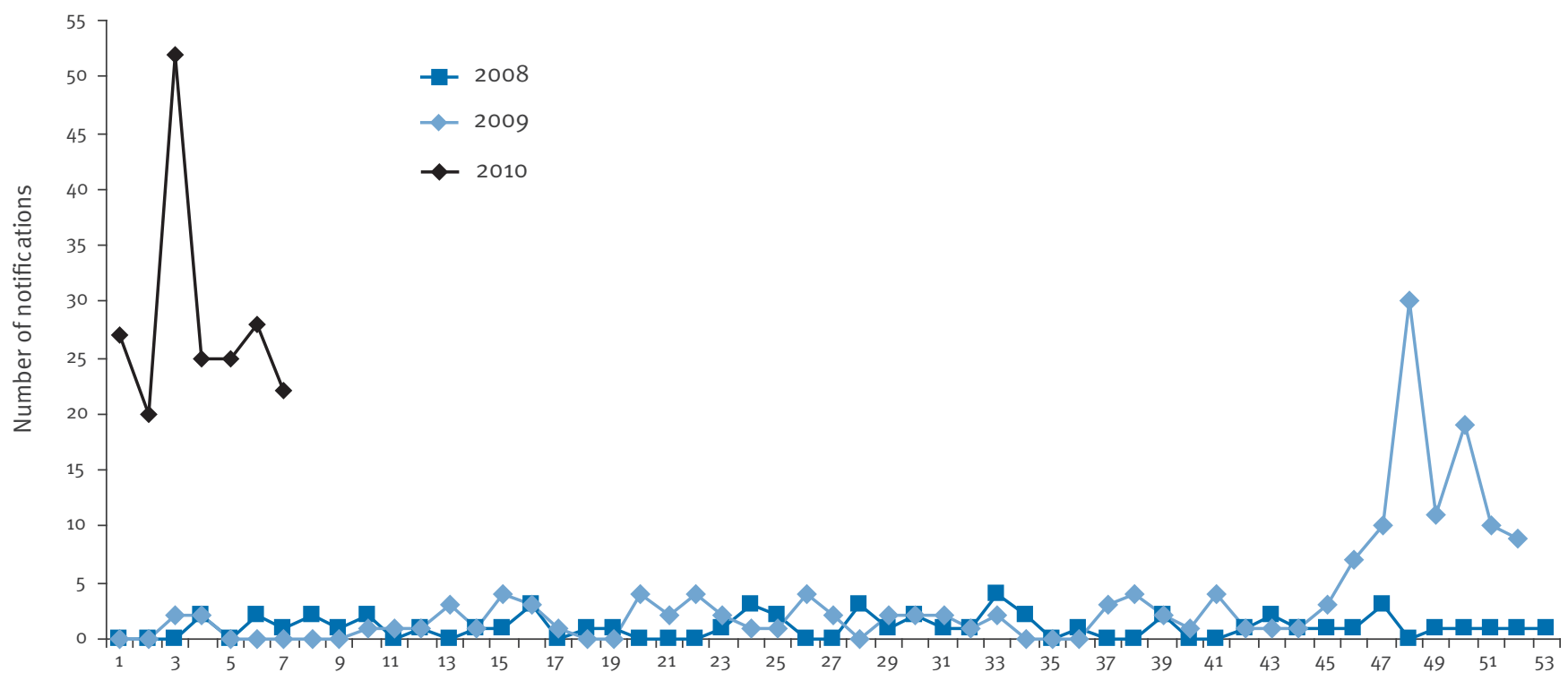

Week of notification 
while one had no case classification specified. Measles notifications and crude measles incidence rates by HSE Area are shown in Figure 1 . The majority (89\%) of cases were under 20 years of age with the largest number of cases (21\%) in the age group of 1-2-year-olds (Figure 3 ).

The highest incidence rate was seen in those younger than one year (Figure 4). Of the 320 cases notified, 174 (54\%) were male and 144 (45\%) were female, while sex was not recorded for two cases (1\%).

Of the 320 notified measles cases, 206 (64\%) were unvaccinated; 45 (14\%) were reported to have had one dose of MMR; six (2\%) were reported to have had two doses of MMR and for $63(20 \%)$ the number of doses of MMR was unknown/not reported. Vaccination dates were reported for one of the six cases with two MMR doses and for 36 cases with one MMR dose (nine of these were vaccinated less than nine days before onset of illness and were probably incubating measles at the time of vaccination).

Of the 320 cases, 115 (36\%) were hospitalised and 162 ( $51 \%)$ were not hospitalised, while hospitalisation status was unknown/not reported for 43 (13\%). Length of

\section{FIGURE 3}

Measles notifications by age group and case classification, Ireland, week 31, 2009 to week 7, 2010

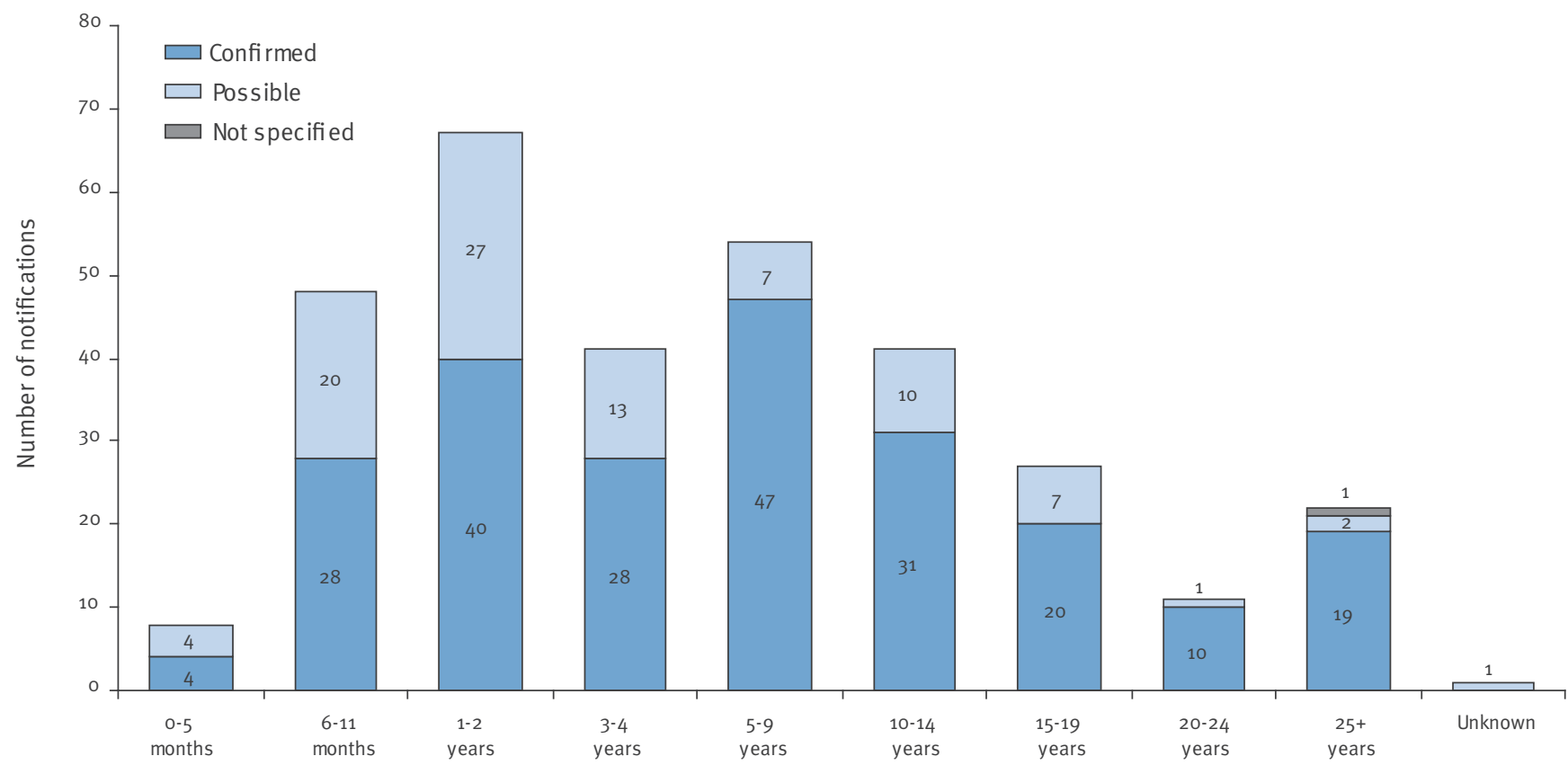

Age group

\section{FIGURE 4}

Age specific incidence rates of measles notifications, Ireland, week 31, 2009 to week 7, 2010

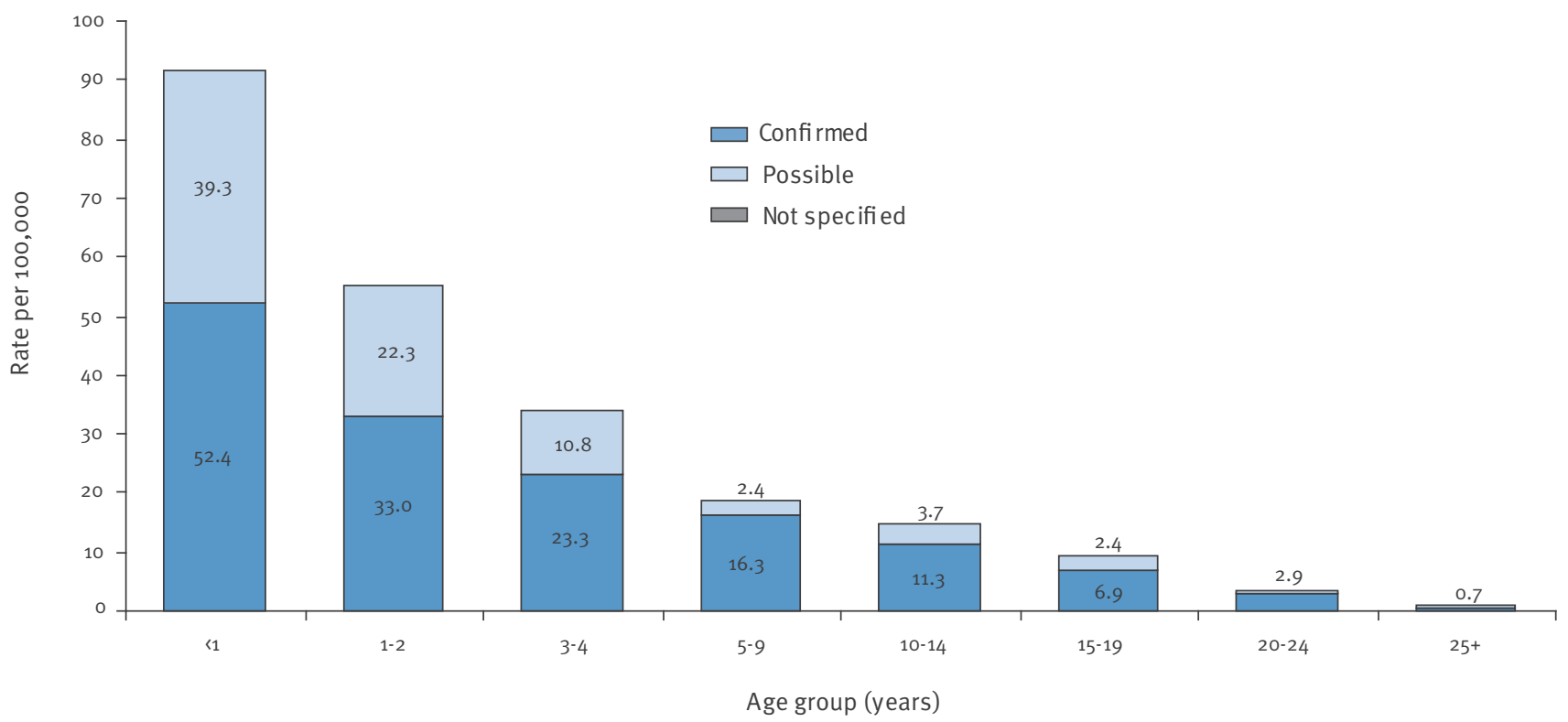


hospitalisation was reported for 73 cases; the median duration of stay was three days (range one to 11 days).

Complications reported included pneumonia $(n=16)$, ear infection/otitis media $(n=4)$, dehydration $(n=2)$, chest infection $(n=1)$, dehydration, nausea and vomiting $(n=1)$, pharyngitis $(n=1)$, pneumothorax $(n=1)$, seizures $(n=1)$ and tonsillitis $(n=1)$.

\section{National outbreak control team}

At the start of the outbreak, a national outbreak control team was convened, which includes health professionals from the departments of public health in the HSE Areas, HSE-Health Protection Surveillance Centre, HSENational Immunisation Office, HSE Population Health, HSE Social Inclusion, the Institute of Obstetricians and Gynaecologists, the National Virus Reference Laboratory and the field of Paediatric Infectious Disease. This group agreed public health strategies (vaccination and management of cases and close contacts, awareness-raising among clinicians and community) to control the outbreak at national and local level. Some of the guidance and strategies recommended by the outbreak control team are outlined here.

\section{General guidance}

All children should be vaccinated at 12 months and 4-5 years, as per the routine childhood immunisation schedule. All children who have not had two MMR vaccines by the age of five years should be offered vaccination opportunistically. Control measures for measles outbreaks were distributed to various settings and healthcare staff and are available on the HPSC website [5].

\section{Traveller community}

All Traveller children who have not had two documented doses of MMR are recommended MMR. All Traveller children aged 6-11 months during the current outbreak are recommended MMR (these children are also recommended MMR again at 12 months and at 4-5 years, as per the normal childhood immunisation schedule). Traveller children who have received MMR are recommended $\mathrm{MMR}_{2}$; $\mathrm{MMR}_{2}$ may be given one month after the first dose (if children under 18 months of age are given $M M R_{2}$ less than three months after $M M R_{1}$, these children need a third dose at 4-5 years of age). MMR vaccine clinics and GP sites were organised to provide MMR to the Traveller community. A subgroup of the outbreak control team was established to liaise with social inclusion groups and non-governmental organisations to find ways to increase vaccination among ethnic minority groups.

\section{Contacts of cases}

MMR given within 72 hours of exposure may prevent infection. Children in outbreak situations who have received $M M R_{1}$ are recommended $M M R_{2} ; M M R_{2}$ may be given one month after the first dose.

\section{Healthcare staff}

All healthcare staff born since 1978 should either be immune to measles or have had two documented doses of MMR. Healthcare staff born before 1978 should be offered MMR if they are considered at high risk of exposure. Guidance on preventing measles transmission in healthcare settings (such as rapid triage and case isolation in addition to vaccination) was distributed to healthcare staff and is available on the HPSC website [5].

\section{MMR catch-up campaign}

An MMR catch-up campaign is planned for school children aged approximately four to 15 years (older school children aged were previously targeted in an MMR campaign in 2009).

\section{Conclusion}

As $29 \%$ of cases in this outbreak are currently classified as possible cases, and although the laboratory results of some of these cases are pending at the time of writing, there is a continued need to strengthen measles surveillance in Ireland and ensure rigorous case investigation and laboratory confirmation of all suspected measles cases. This outbreak highlights once again the need for an MMR vaccine uptake of at least $95 \%$ to prevent measles outbreaks and the importance of increasing coverage in all groups, in particular those groups who are hard to access. The simultaneous occurrence of the 2009 influenza $A\left(\mathrm{H}_{1} \mathrm{~N}_{1}\right)$ pandemic and the ensuing pandemic vaccination programme has put enormous pressure on vaccination teams trying to address MMR defaulters at the same time. There is a concern that this current outbreak may develop into a large outbreak similar to the one that occurred in 2000 [2].

Acknowledgements

HPSC wish to sincerely thank everyone who contributed to measles surveillance in Ireland.

The members of the national measles outbreak control team were (in alphabetical order):

A Breslin, A Clarke, B Corcoran, J Connell, S Cotter, P Finnegan, R Fitzgerald, S Gee, P Gavin, J Heslin, K Kelleher, R Kiernan, P Mc Keown, H Murray, E O’Connell, M O'Connell, D O'Flanagan, A O’Malley, L O’Neill, H Pelly, F Ryan, M Ward

\section{References}

1. Immunisation Uptake Statistics. Quarterly Reports. Dublin, Health Protection Surveillance Centre. Available from: http:// www.hpsc.ie/hpsc/A-Z/VaccinePreventable/Vaccination/ ImmunisationUptakeStatistics/QuarterlyReports/

2. Annual Report of the National Disease Surveillance Centre, 2000. Dublin, Health Protection Surveillance Centre; 2001. ISSN:1649-0436. Available from: http://www.hpsc.ie/hpsc/ AboutHPSC/AnnualReports/File,520,en.pdf

3. Case Definitions for Notifiable Diseases. Infectious Diseases (Amendment) (No. 3) Regulations 2003 (SI No. 707 of 2003). Dublin, Health Protection Surveillance Centre. Available at http://www.ndsc.ie/hpsc/NotifiableDiseases/CaseDefinitions/ File,823,en.pdf

4. Factsheets - Irish Travellers. Pavee Point Travellers Centre. [Internet]. Available from: http://www.paveepoint.ie/fs irish a.html

5. Measles. Guidance documents. Dublin, Health Protection Surveillance Centre. Available from: http://www.hpsc.ie/ hpsc/A-Z/VaccinePreventable/Measles/Guidance/ 